\title{
Effect of bimodularity on frequency response of cylindrical panels using Galerkin time domain approach
}

\author{
KALLOL KHAN, BADRI PRASAD PATEL* and \\ YOGENDRA NATH
}

Department of Applied Mechanics, Indian Institute of Technology Delhi, Hauz Khas, New Delhi 110016

e-mail: kallol_rec@yahoo.co.in,badripatel@hotmail.com,

nathyogendra@hotmail.com

MS received 16 September 2009; revised 14 August 2010; accepted 30 August 2010

\begin{abstract}
The forced vibration analysis of bimodulus material laminated structures is a challenging problem due to non-smooth nonlinear nature of governing equations. The most commonly used direct time integration schemes show numerical instability and do not predict steady state response except for limited number of cases without considering in-plane inertia. This is due to the sudden change of restoring force from positive/negative half cycle to negative/positive half cycle exciting higher modes/harmonics at every instant of a cycle change leading to numerical instability in the time marching scheme. In the present work, Galerkin time domain approach is successfully used for the forced vibration analysis of bimodular cylindrical panels. The effect of bimodularity ratio on the frequency response of cylindrical panels for few typical geometrical and lamination parameters is studied for the first time. It is found that the positive half cycle amplitude is greater than the negative half cycle amplitude for $E_{2 t} / E_{2 c}<1$ and is smaller for $E_{2 t} / E_{2 c}>1$. Further, the percentage difference of positive and negative half cycle amplitudes decreases with the increase in $E_{2 t} / E_{2 c}$. The stresses under dynamic loading are different for positive and negative half of a vibration cycle.
\end{abstract}

Keywords. Bimodularity; frequency response; time domain Galerkin; positive/negative half cycles.

\section{Introduction}

Bimodularity, the different behaviour of material in tension and compression, is an inherent characteristic of almost all fibre reinforced composites. The actual stress strain behaviour of such materials is nonlinear which can be approximated by bilinear model with slope discontinuity at the origin as shown in figure 1. For certain fibre reinforced composites, the

*For correspondence 


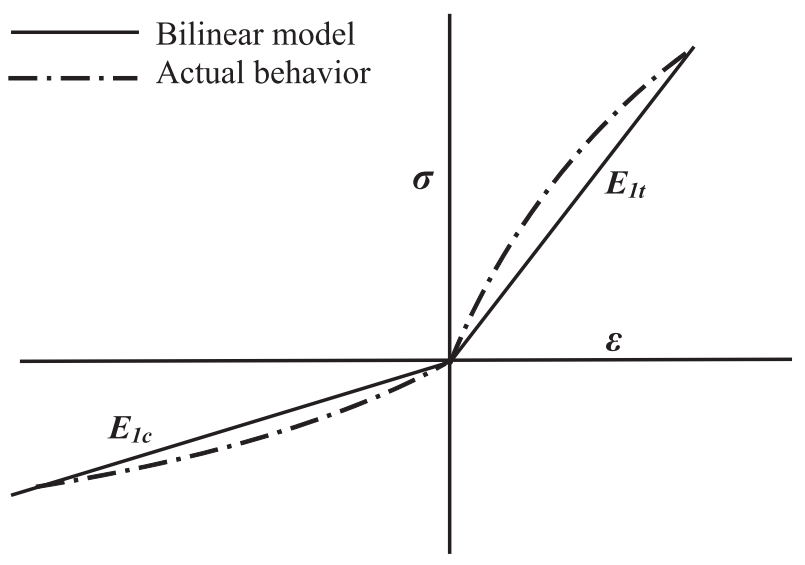

Figure 1. Typical stress-strain relation of bimodulus material.

bimodularity ratio $\left(E_{1 t} / E_{1 c}\right)$ is very high e.g. unidirectional glass/epoxy composites have compression moduli $20 \%$ lesser than the tension moduli, boron/epoxy laminates have compression moduli about 15-20\% greater than the tension moduli and graphite/epoxy laminates have tension moduli up to $40 \%$ greater than the compression moduli, carbon fibre has tensile moduli about 2 times of the compression moduli (Venkatrao et al 2008), aramid-rubber has tensile moduli about 300 times of the compression moduli (Bert et al 1981).

The dynamic analysis of laminated composite structures considering bimodularity has received the attention of few researchers due to non-smooth nonlinear nature of problem. The free vibration analysis of bimodular material cross-ply laminated rectangular plates is carried out by Bert et al (1981), Doong \& Chen (1985), Doong \& Fung (1988). Patel et al (2005a) have carried out the free vibration analysis of angle-ply laminated bimodulus thick rectangular plates. The free vibration analysis of cross- and angle-ply cylindrical panels is carried out by Bert \& Kumar (1982) and Khan et al (2007, 2009a), respectively. The free vibration of cross-ply conical panels is carried out by Khan et al (2008). The transient response of bimodular rectangular plates is studied by Reddy (1982) and Patel et al (2005b) wherein the investigation/analysis is limited to first cycle only.

The work on frequency response of bimodular structures is scarce in open literature (Khan et al 2009b). This may be attributed to the numerical instability due to non-smooth nonlinear nature of equations of motion while integrating for sufficient number of cycles to reach the steady state. The sudden change of restoring force from positive/negative half cycle to negative/positive half cycle excites higher modes/harmonics at every instant of cycle change leading to numerical instability in the time marching scheme. The direct time integration schemes can be applied for the frequency response analysis of bimodular structures only for cases without considering in-plane inertia and having smaller difference in the positive and negative half cycle stiffnesses.

To overcome this difficulty, a methodology based on time domain Galerkin approach has been proposed to find frequency response of bimodular material laminated panels by Khan et al (2009b). The application of the method for cross-ply aramid-rubber $\left(E_{1 t} / E_{1 c}=300\right)$ laminated cylindrical panels has been demonstrated. To the best of the authors' knowledge, the effect of bimodularity ratio $\left(E_{2 t} / E_{2 c}\right)$ on the frequency response of cross- and angleply laminated structures is not dealt in the open literature. Due to the evident importance of frequency response for the design of laminated structures, the present study is undertaken for cross- and angle-ply bimodular material laminated cylindrical panels. 


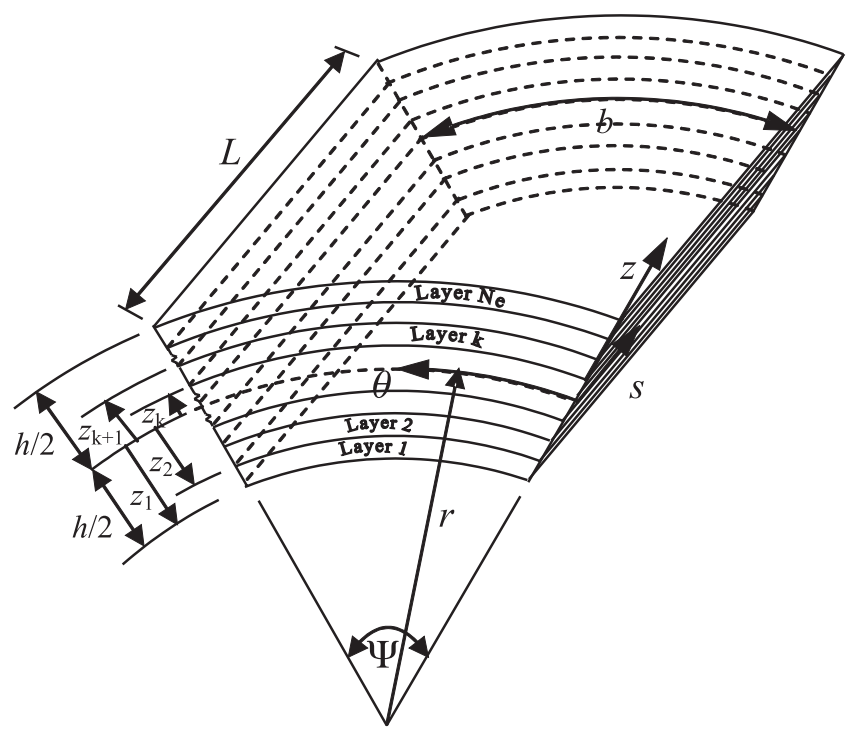

Figure 2. Geometry and coordinate system of a laminated cylindrical panel.

\section{Formulation}

The geometry and coordinate system of a cylindrical panel with total thickness $h$, radius $r$, meridional length $L$, circumferential length $b$ and sector angle $\Psi$ are shown in figure 2 . The displacement field $(u, v, w)$ at a point $(s, \theta, z)$ is expressed as functions of middle surface displacements $u_{0}, v_{0}, w_{0}$ and independent rotations $\beta_{s}$ and $\beta_{\theta}$ of the meridional and hoop sections, respectively, as:

$$
\begin{aligned}
& u(s, \theta, z, t)=u_{0}(s, \theta, t)+z \beta_{s}(s, \theta, t) \\
& v(s, \theta, z, t)=v_{0}(s, \theta, t)+z \beta_{\theta}(s, \theta, t) \\
& w(s, \theta, z, t)=w_{0}(s, \theta, t) .
\end{aligned}
$$

The strain-displacement relations are based on Sanders type of kinematic approximations (Kraus 1976): (i) small strains and (ii) thin shell $(z / r \ll 1)$ such that $1+z / r \approx 1$. Transverse shear deformation is important due to higher $E / G$ ratio for composites.

The strain vector can be written as:

$$
\{\boldsymbol{\varepsilon}\}=\left\{\begin{array}{c}
\varepsilon_{s s} \\
\varepsilon_{\theta \theta} \\
\gamma_{s \theta} \\
\gamma_{s z} \\
\gamma_{\theta z}
\end{array}\right\}=\left\{\begin{array}{c}
\varepsilon_{p} \\
0
\end{array}\right\}+\left\{\begin{array}{c}
z \varepsilon_{b} \\
\varepsilon_{s}
\end{array}\right\} .
$$


The vectors $\left\{\varepsilon_{p}\right\},\left\{\varepsilon_{b}\right\}$ and $\left\{\varepsilon_{s}\right\}$ represent mid-surface membrane, bending and transverse shear strains, respectively, and are defined as:

$$
\left\{\varepsilon_{p}\right\}=\left\{\begin{array}{c}
\frac{\partial u_{0}}{\partial s} \\
\frac{\partial v_{0}}{r \partial \theta}+\frac{w_{0}}{r} \\
\frac{\partial u_{0}}{r \partial \theta}+\frac{\partial v_{0}}{\partial s}
\end{array}\right\},\left\{\varepsilon_{b}\right\}=\left\{\begin{array}{c}
\frac{\partial \beta_{s}}{\partial s} \\
\frac{\partial \beta_{\theta}}{r \partial \theta} \\
\frac{\partial \beta_{s}}{r \partial \theta}+\frac{\partial \beta_{\theta}}{\partial s}
\end{array}\right\},\left\{\varepsilon_{s}\right\}=\left\{\begin{array}{c}
\beta_{s}+\frac{\partial w_{0}}{\partial s} \\
\beta_{\theta}+\frac{\partial w_{0}}{r \partial \theta}-\frac{v_{0}}{r}
\end{array}\right\} .
$$

Based on the fibre direction strain governed model, the constitutive relation of a bimodulus laminated material can be written as (Bert 1977):

$$
\left\{\boldsymbol{\sigma}^{\boldsymbol{k}}\right\}=\left\{\begin{array}{c}
\sigma_{s s}^{k} \\
\sigma_{\theta \theta}^{k} \\
\tau_{s \theta}^{k} \\
\tau_{s z}^{k} \\
\tau_{\theta z}^{k}
\end{array}\right\}=\left[\begin{array}{ccccc}
\bar{Q}_{11 k l} & \bar{Q}_{12 k l} & \bar{Q}_{16 k l} & 0 & 0 \\
\bar{Q}_{12 k l} & \bar{Q}_{22 k l} & \bar{Q}_{26 k l} & 0 & 0 \\
\bar{Q}_{16 k l} & \bar{Q}_{26 k l} & \bar{Q}_{66 k l} & 0 & 0 \\
0 & 0 & 0 & \bar{Q}_{44 k l} & \bar{Q}_{45 k l} \\
0 & 0 & 0 & \bar{Q}_{45 k l} & \bar{Q}_{55 k l}
\end{array}\right]\left\{\begin{array}{c}
\varepsilon_{s s} \\
\varepsilon_{\theta \theta} \\
\gamma_{s \theta} \\
\gamma_{s z} \\
\gamma_{\theta z}
\end{array}\right\}=\left[\bar{Q}_{i j k l}\right]\{\boldsymbol{\varepsilon}\},
$$

where $\bar{Q}_{i j k l}$ are transformed stiffness coefficients and $k$ is layer number, $l=1$ denotes the properties associated with fibre direction tension and $l=2$ denotes the properties associated with fibre direction compression.

The kinetic energy of the shell is expressed as:

$$
T(\delta)=\frac{1}{2} \iint\left[\sum_{k=1}^{N} \int_{h_{k}}^{z_{z k+1}} \rho_{k}\left\{\dot{u}_{k} \dot{v}_{k} \dot{w}_{k}\right\}\left\{\dot{u}_{k} \dot{v}_{k} \dot{w}_{k}\right\}^{T} d z\right] r d s d \theta,
$$

where $\rho_{k}$ is the mass density of the $k^{\text {th }}$ layer; $\mathrm{z} k, h_{k+1}$ are the $z$-coordinates of the bottom and top surfaces of the $k^{\text {th }}$ layer and $\{\delta\}=\left\{\delta_{1} \delta_{2} \ldots \delta_{n}\right\}^{T}$ is the vector of degrees of freedom.

The potential energy of the shell is given by:

$$
U(\delta)=\frac{1}{2} \iint\left[\sum_{k=1}^{N} \int_{h_{k}}^{z_{z k+1}}\{\sigma\}^{T}\{\varepsilon\} d z\right] r d s d \theta-\iint q w_{0} r d s d \theta,
$$

where $q$ is distributed force.

Analysis is carried out using a $C^{0}$ eight-noded serendipity quadrilateral shear flexible shell element with 5 nodal degrees of freedom $\left(u_{0}, v_{0}, w_{0}, \beta_{s}, \beta_{\theta}\right)$ developed based on the field consistency approach (Prathap et al 1988).

The governing equations of motion, considering dissipative forces, take the form:

$$
[\mathbf{M}]\{\ddot{\boldsymbol{\delta}}\}+[\mathbf{C}]\{\dot{\boldsymbol{\delta}}\}+[\mathbf{K}]\{\boldsymbol{\delta}\}=\{\mathbf{F}\},
$$

where $[\mathbf{M}],[\mathbf{K}]$ and $[\mathbf{C}]$ are global mass, stiffness and damping matrices, and $\{\mathbf{F}\}$ is consistent global load vector. The damping matrix is taken proportional to mass matrix as: $[\mathbf{C}]=\beta[\mathbf{M}]$; $\beta=2 \xi \omega$ where $\xi$ is modal damping factor and $\omega$ is natural frequency.

Assuming solution $\{\delta\}=\{\bar{\delta}\} e^{i \omega t}$ for undamped free vibration, equation (7) becomes:

$$
[\mathbf{K}]\{\overline{\boldsymbol{\delta}}\}=\omega^{2}[\mathbf{M}]\{\overline{\boldsymbol{\delta}}\} .
$$

Free vibration frequencies and corresponding modal vectors are extracted using iterative eigenvalue approach. 


\section{Solution method}

For the forced response analysis, the solution of equation (7) is obtained using time domain Galerkin approach proposed by Khan et al (2009b). For the sake of completeness, the method is briefly discussed here. Few results are compared with those obtained using Newmark's constant-average acceleration scheme (Bathe 1996). It is found that for bimodulus laminates with significant difference in the positive and negative half cycle free vibration frequencies, the direct time integration approach fails to predict steady state response.

In Galerkin method, the solution is assumed as:

$$
\{\boldsymbol{\delta}\}=\left\{\boldsymbol{\delta}_{\mathbf{0}}\right\}+\sum_{i=1}^{m}\left\{\boldsymbol{\delta}_{\mathbf{c i}}\right\} \operatorname{Cos} i \omega_{F} t+\left\{\boldsymbol{\delta}_{\mathbf{s i}}\right\} \operatorname{Sin} i \omega_{F} t,
$$

where $\omega_{F}$ is forcing frequency and $t$ is time.

Substituting equation (9) in equation (7), the residual vector $\{\mathbf{R}\}$ is written as:

$$
\begin{aligned}
\{\mathbf{R}\}= & -\omega_{F}^{2}[\mathbf{M}] \sum_{i=1}^{m} i^{2}\left(\left\{\boldsymbol{\delta}_{\mathbf{c i}}\right\} \operatorname{Cos} i \omega_{F} t+\left\{\boldsymbol{\delta}_{\mathbf{s i}}\right\} \operatorname{Sin} i \omega_{F} t\right) \\
& -\omega_{F}[\mathbf{C}] \sum_{i=1}^{m} i\left(\left\{\boldsymbol{\delta}_{\mathbf{c i}}\right\} \operatorname{Sin} i \omega_{F} t-\left\{\boldsymbol{\delta}_{\mathbf{s i}}\right\} \operatorname{Cos} i \omega_{F} t\right)+[\mathbf{K}]\left\{\boldsymbol{\delta}_{\mathbf{0}}\right\} \\
& +[\mathbf{K}] \sum_{i=1}^{m}\left(\left\{\boldsymbol{\delta}_{\mathbf{c i}}\right\} \operatorname{Cos} i \omega_{F} t+\left\{\boldsymbol{\delta}_{\mathbf{s i}}\right\} \operatorname{Sin} i \omega_{F} t\right)-\{\mathbf{F}\}
\end{aligned}
$$

To generate the $(2 m+1) n$ equations in terms of $(2 m+1) n$ unknowns $\left(\left\{\boldsymbol{\delta}_{\mathbf{0}}\right\},\left\{\boldsymbol{\delta}_{\mathbf{c i}}\right\},\left\{\boldsymbol{\delta}_{\mathbf{s i}}\right\}\right.$, $i=1,2, \ldots, m)$, the weighted integral of residual $\{\mathbf{R}\}$ over one time period is equated to zero for weighting functions: $1, \operatorname{Cos} i \omega_{F} t, \operatorname{Sin} i \omega_{F} t, i=1,2, \ldots, m$. For each weighting function (say $\operatorname{Cos} i \omega_{F} t$ ), the integration with respect to time is performed piece-wise as follows:

$$
\int_{0}^{t_{1}}\{\mathbf{R}\} \operatorname{Cos} i \omega_{F} t d t+\int_{t_{1}}^{t_{2}}\{\mathbf{R}\} \operatorname{Cos} i \omega_{F} t d t+\int_{t_{2}}^{2 \pi / \omega_{F}}\{\mathbf{R}\} \operatorname{Cos} i \omega_{F} t d t=0 .
$$

Here $t_{1}$ and $t_{2}$ are time instants within a cycle where response changes from positive/negative half cycle to negative/positive half cycle (see figure 3 ). It may be noted that $\{\mathbf{R}\}$ is different
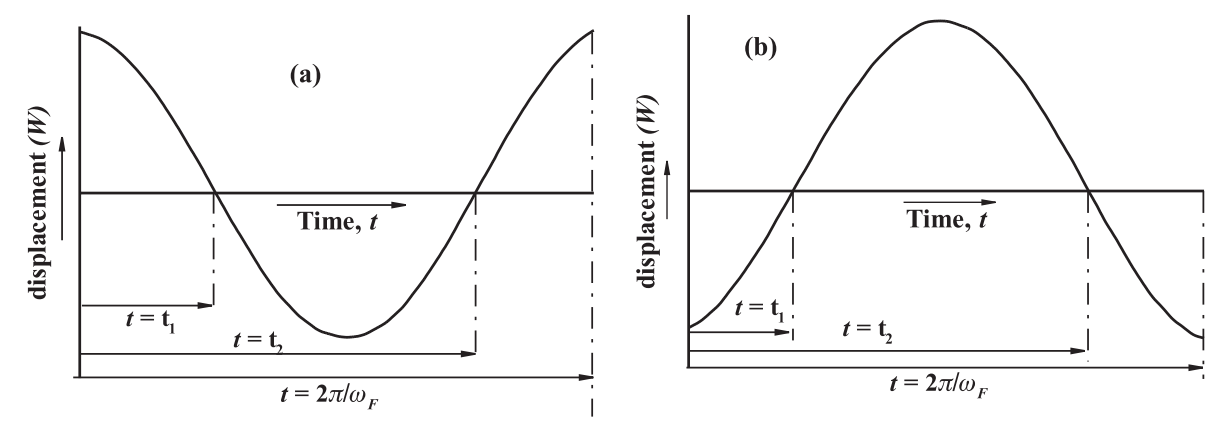

Figure 3. Response in a typical vibration cycle of bimodular panel. 
for positive and negative half cycles since the stiffness matrix $[\mathbf{K}]$ is different for positive (say $\left[\mathbf{K}_{\mathbf{1}}\right]$ ) and negative (say $\left[\mathbf{K}_{\mathbf{2}}\right]$ ) half cycles.

The final set of equations, considering all the $(2 m+1)$ weighting functions, is written as:

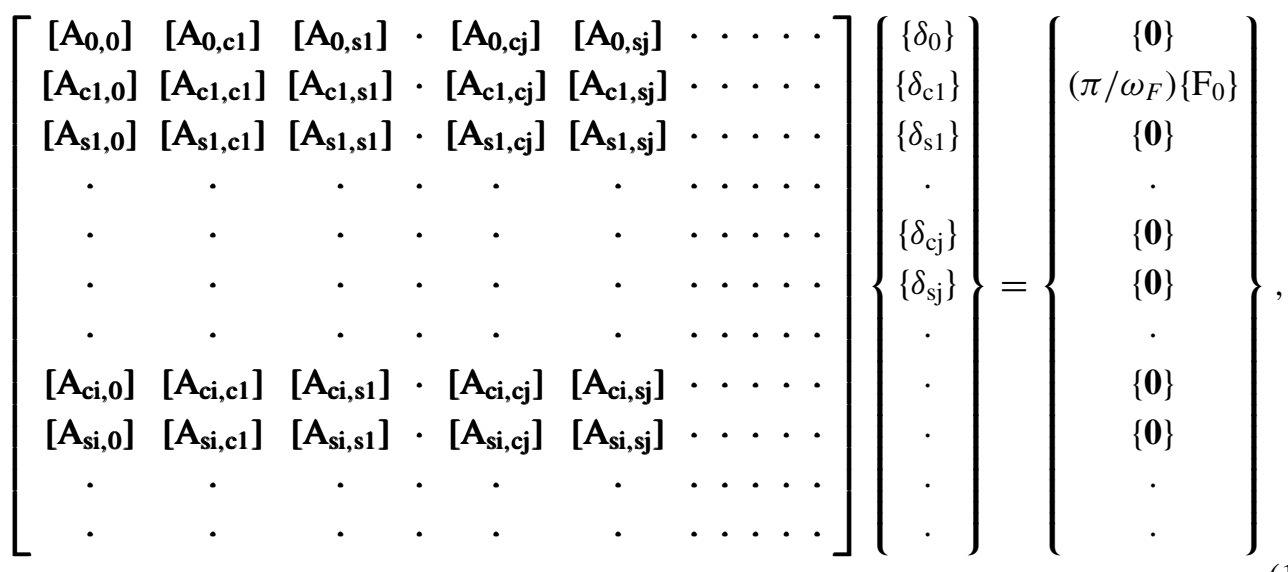

where coefficient sub matrices of equation (12), for motion from 0 to $t_{1}$ and $t_{2}$ to $2 \pi / \omega_{F}$ corresponding to positive half cycle and that from $t_{1}$ to $t_{2}$ corresponding to negative half cycle, are expressed as:

$$
\begin{aligned}
& {\left[\mathbf{A}_{\mathbf{0}, \mathbf{0}}\right]=\left[\mathbf{K}_{\mathbf{1}}\right]\left(t_{1}-t_{2}+2 \pi / \omega_{F}\right)+\left[\mathbf{K}_{\mathbf{2}}\right]\left(t_{2}-t_{1}\right) ;} \\
& {\left[\mathbf{A}_{\mathbf{0}, \mathbf{c j}}\right]=\left[\left[\mathbf{K}_{\mathbf{1}}\right]-\left[\mathbf{K}_{\mathbf{2}}\right]\right]\left(\operatorname{Sin} j \omega_{F} t_{1}-\operatorname{Sin} j \omega_{F} t_{2}\right) /\left(j \omega_{F}\right), \quad j=1, \ldots, m ;} \\
& {\left[\mathbf{A}_{\mathbf{0}, \mathbf{s j}}\right]=\left[\left[\mathbf{K}_{\mathbf{1}}\right]-\left[\mathbf{K}_{\mathbf{2}}\right]\right]\left(\operatorname{Cos} j \omega_{F} t_{2}-\operatorname{Cos} j \omega_{F} t_{1}\right) /\left(j \omega_{F}\right), \quad j=1, \ldots, m ;} \\
& {\left[\mathbf{A}_{\mathbf{c i}, \mathbf{0}}\right]=\left[\left[\mathbf{K}_{\mathbf{1}}\right]-\left[\mathbf{K}_{\mathbf{2}}\right]\right]\left(\operatorname{Sin} i \omega_{F} t_{1}-\operatorname{Sin} i \omega_{F} t_{2}\right) /\left(i \omega_{F}\right), \quad i=1, \ldots, m ;} \\
& {\left[\mathbf{A}_{\mathbf{s i}, \mathbf{0}}\right]=\left[\left[\mathbf{K}_{\mathbf{1}}\right]-\left[\mathbf{K}_{\mathbf{2}}\right]\right]\left(\operatorname{Cos} i \omega_{F} t_{2}-\operatorname{Cos} i \omega_{F} t_{1}\right) /\left(i \omega_{F}\right), \quad i=1, \ldots, m ;}
\end{aligned}
$$

For $i \neq j$ :

$$
\begin{aligned}
{\left[\mathbf{A}_{\mathbf{c i}, \mathbf{j}}\right]=} & {\left[\left[\mathbf{K}_{\mathbf{1}}\right]-\left[\mathbf{K}_{\mathbf{2}}\right]\right]\left(\left(\operatorname{Sin}(i+j) \omega_{F} t_{1}-\operatorname{Sin}(i+j) \omega_{F} t_{2}\right) /(i+j)\right.} \\
& \left.+\left(\operatorname{Sin}(i-j) \omega_{F} t_{1}-\operatorname{Sin}(i-j) \omega_{F} t_{2}\right) /(i-j) /\left(2 \omega_{F}\right)\right) ; \\
{\left[\mathbf{A}_{\mathbf{c i}, \mathbf{s}}\right]=} & {\left[\left[\mathbf{K}_{\mathbf{1}}\right]-\left[\mathbf{K}_{\mathbf{2}}\right]\right]\left(\left(\operatorname{Cos}(i+j) \omega_{F} t_{1}-\operatorname{Cos}(i+j) \omega_{F} t_{2}\right) /(i+j)\right.} \\
& \left.+\left(\operatorname{Cos}(i-j) \omega_{F} t_{1}-\operatorname{Cos}(i-j) \omega_{F} t_{2}\right) /(i-j) /\left(2 \omega_{F}\right)\right) ; \\
{\left[\mathbf{A}_{\mathbf{s i}, \mathbf{j}}\right]=} & {\left[\left[\mathbf{K}_{\mathbf{1}}\right]-\left[\mathbf{K}_{\mathbf{2}}\right]\right]\left(\left(\operatorname{Cos}(i+j) \omega_{F} t_{2}-\operatorname{Cos}(i+j) \omega_{F} t_{1}\right) /(i+j)\right.} \\
& \left.+\left(\operatorname{Cos}(i-j) \omega_{F} t_{2}-\operatorname{Cos}(i-j) \omega_{F} t_{1}\right) /(i-j) /\left(2 \omega_{F}\right)\right) ; \\
{\left[\mathbf{A}_{\mathbf{s i}, \mathbf{s}}\right]=} & {\left[\left[\mathbf{K}_{\mathbf{1}}\right]-\left[\mathbf{K}_{\mathbf{2}}\right]\right]\left(\left(\operatorname{Sin}(i+j) \omega_{F} t_{2}-\operatorname{Sin}(i+j) \omega_{F} t_{1}\right) /(i+j)\right.} \\
& \left.+\left(\operatorname{Sin}(i-j) \omega_{F} t_{1}-\operatorname{Sin}(i-j) \omega_{F} t_{2}\right) /(i-j) /\left(2 \omega_{F}\right)\right) ;
\end{aligned}
$$


For $i=j$ :

$$
\begin{aligned}
{\left[\mathbf{A}_{\mathbf{c i}, \mathbf{c} j}\right]=} & {\left[\left[\mathbf{K}_{1}\right]-\left[\mathbf{K}_{2}\right]\right]\left(\left(t_{1}-t_{2}\right) / 2+\left(\operatorname{Sin} 2 i \omega_{F} t_{1}-\operatorname{Sin} 2 i \omega_{F} t_{2}\right) /\left(4 i \omega_{F}\right)\right) } \\
& +\left[\mathbf{K}_{\mathbf{1}}\right]\left(\pi / \omega_{F}\right)-[\mathbf{M}] \pi i^{2} \omega_{F} ; \\
{\left[\mathbf{A}_{\mathbf{c i}, \mathbf{s j}}\right]=} & {\left[\left[\mathbf{K}_{\mathbf{1}}\right]-\left[\mathbf{K}_{\mathbf{2}}\right]\right]\left(\operatorname{Cos} 2 i \omega_{F} t_{2}-\operatorname{Cos} 2 i \omega_{F} t_{1}\right) /\left(4 i \omega_{F}\right)+[\mathbf{C}] i \pi ; } \\
{\left[\mathbf{A}_{\mathbf{s i}, \mathbf{j} j}\right]=} & {\left[\left[\mathbf{K}_{1}\right]-\left[\mathbf{K}_{2}\right]\right]\left(\operatorname{Cos} 2 i \omega_{F} t_{2}-\operatorname{Cos} 2 i \omega_{F} t_{1}\right) /\left(4 i \omega_{F}\right)-[\mathbf{C}] i \pi ; } \\
{\left[\mathbf{A}_{\mathbf{s i}, \mathbf{s j}}\right]=} & {\left[\left[\mathbf{K}_{\mathbf{1}}\right]-\left[\mathbf{K}_{\mathbf{2}}\right]\right]\left(\left(t_{1}-t_{2}\right) / 2-\left(\operatorname{Sin} 2 i \omega_{F} t_{1}-\operatorname{Sin} 2 i \omega_{F} t_{2}\right) /\left(4 i \omega_{F}\right)\right) } \\
& +\left[\mathbf{K}_{\mathbf{1}}\right]\left(\pi / \omega_{F}\right)-[\mathbf{M}] \pi i^{2} \omega_{F} .
\end{aligned}
$$

In the above expressions, [ $\left.\mathbf{K}_{\mathbf{1}}\right]$ and $\left[\mathbf{K}_{\mathbf{2}}\right]$ are interchanged if motion from 0 to $t_{1}$ and $t_{2}$ to $2 \pi / \omega_{F}$ corresponds to negative half cycle and that from $t_{1}$ to $t_{2}$ corresponds to positive half cycle.

Since $t_{1}$ and $t_{2}$ are not known a priori, two additional equations are required. These equations are obtained by equating the transverse displacement at an anti-node point of a mode (frequency response is studied in the neighbourhood of a free vibration frequency) to zero at $t_{1}$ and $t_{2}$ as follows:

$$
\begin{aligned}
& w_{00}+\sum_{i=1}^{m} w_{0 c i} \operatorname{Cos} i \omega_{F} t_{1}+w_{0 s i} \operatorname{Sin} i \omega_{F} t_{1}=0 \\
& w_{00}+\sum_{i=1}^{m} w_{0 c i} \operatorname{Cos} i \omega_{F} t_{2}+w_{0 s i} \operatorname{Sin} i \omega_{F} t_{2}=0 .
\end{aligned}
$$

Equations (12)-(14) constitute $(2 m+1) n+2$ nonlinear (due to presence of $t_{1}$ and $t_{2}$ ) equations in $(2 m+1) n+2$ unknowns. The solution of these equations is obtained using NewtonRaphson iterative technique.

The coefficient matrix of equation (12) is unsymmetric and sparse but the non-zero coefficients are scattered throughout. It may be noted that the matrices in equation (7) are symmetric and banded with semi-bandwidth $\left(n_{b}\right)$ being much smaller than the total number of unknowns $(n)$. Equation (12) is rearranged so that the rearranged equations are banded with semi-bandwidth equal to $m$ times $n_{b}$.

While solving equations (12)-(14), firstly the incremental displacement vectors $\left(\left\{\Delta \delta_{0}\right\}\right.$, $\left.\left\{\Delta \delta_{\mathbf{c i}}\right\},\left\{\Delta \delta_{\mathbf{s i}}\right\}, i=1,2,3, \ldots, m\right)$ are expressed in terms of $\Delta t_{1}$ and $\Delta t_{2}$. Using this solution and incremental forms of equations (13) and (14), $\Delta t_{1}$ and $\Delta t_{2}$ are obtained and then, the incremental displacement vectors. To start the solution, forcing frequency ratio $\left(\omega_{F} / \omega, \omega\right.$ is fundamental free vibration frequency) is taken as 0.9 . At this starting point, displacement vectors are initialized to zero, and $t_{1}$ and $t_{2}$ are assumed as $\pi /\left(2 \omega_{F}\right)$ and $3 \pi /\left(2 \omega_{F}\right)$, respectively. With these starting values, the iteration is continued till the solution converges to the specified convergence tolerance (each incremental displacement becomes less than equal to $10^{-7}$ ). Then the forcing frequency is incremented. With the solution of the previous step as starting point, the converged solution for new forcing frequency is obtained. This is repeated till solution up to the desired forcing frequency is obtained.

When $\omega_{F} / \omega$ crosses 1 , the phase lag of response becomes greater than $90^{\circ}$. At forcing frequency ratio greater than one, $t_{1}$ becomes negative if iteration continued from previous solution. For this case, values of $t_{1}$ and $t_{2}$ are reinitialized at the starting iteration so that these lie between 0 and $2 \pi / \omega_{F}$. 


\section{Results and discussion}

The effect of bimodularity ratio on frequency response of cross- and angle-ply bimodular material laminated cylindrical panels is studied under harmonic excitation $\left(q=q_{0} \cos \omega_{\mathrm{F}} t\right)$ in the neighbourhood fundamental mode. Results are presented for the following data:

\subsection{Material properties}

In tension: $E_{1 t} / E_{2 t}=25, E_{2 t}=E_{3 t}, G_{12 t} / E_{2 t}=G_{13 t} / E_{2 t}=0.5, G_{23 t} / E_{2 t}=0.2$, $v_{12 t}=v_{23 t}=v_{13 t}=0.25$.

In compression: $E_{1 c} / E_{2 c}=25, E_{2 c}=E_{3 c}=1 \mathrm{GPa}, G_{12 c} / E_{2 c}=G_{13 c} / E_{2 c}=0 \cdot 5$, $G_{23 c} / E_{2 c}=0 \cdot 2, v_{12 c}=v_{23 c}=v_{13 c}=0 \cdot 25, E_{2 t} / E_{2 c}$ is varied from 0.2 to $2 \cdot 0$, density $\rho=970 \mathrm{Kg} / \mathrm{m}^{3}$.

\subsection{Boundary conditions}

Simply supported:

$$
\begin{array}{ll}
u_{0}=w_{0}=\beta_{s}=0 & \text { at } \theta=0 \text { and } b / r \text { (straight edges). } \\
v_{0}=w_{0}=\beta_{\theta}=0 & \text { at } s=0 \text { and } L \text { (curved edges). }
\end{array}
$$

Clamped: $\quad u_{0}=v_{0}=w_{0}=\beta_{s}=\beta_{\theta}=0 \quad$ at straight $/$ curved edges.

Symmetry:

$$
\begin{array}{ll}
v_{0}=\beta_{\theta}=0 & \text { at } \theta=b / 2 r \\
u_{0}=\beta_{s}=0 & \text { at } s=L / 2 .
\end{array}
$$

The results are presented for two sets of edge conditions: straight edges clamped and curved edges simply supported (CSCS) and all edges clamped (CCCC).

Based on the convergence study, $10 \times 10$ mesh discretization of full model for angle-ply panels and $5 \times 5$ mesh discretization of quarter model for cross-ply panels are found to yield accurate results.

The non-dimensional central transverse displacement $(W)$ presented is defined as: $W=$ $w_{0} h^{3} E_{2 c} / q_{0} L^{4}$. The average frequency $(\omega)$ over a cycle is given by: $\omega=2\left(1 / \omega_{1}+1 / \omega_{2}\right)^{-1}$ where $\omega_{1}$ and $\omega_{2}$ are positive and negative half cycle frequencies, respectively.

Since there are no published results available on frequency response of bimodular material laminated structures, the present formulation is validated for transient response of bimodular cross-ply laminated $\left(0^{\circ} / 90^{\circ}\right)$ simply supported (SSSS) square plate. The results of the comparative study, shown in figure 4, reveal that the present results are in good agreement with those of Reddy (1982).

The response of eight-layered cross-ply $\left(0^{\circ} / 90^{\circ}\right)_{4}$ and angle-ply $\left(45^{\circ} /-45^{\circ}\right)_{4}$ bimodular CSCS panels $\left(L / b=0 \cdot 5, b / h=10, r / h=50, E_{2 t} / E_{2 c}=0 \cdot 2, \omega_{F} / \omega=1\right)$ subjected to uniformly distributed harmonic excitation is obtained using Newmark direct time integration scheme $\left(\Delta t=\pi / 200 \omega_{F}\right)$ with and without considering in-plane inertia. The time versus non-dimensional central transverse displacement responses are shown in figures 5 and 6 for damping factor $\xi=0.01$ and 0.02 , respectively. It can be seen from these figures that the steady state is reached when in-plane inertia is not considered except for cross-ply panel with $\xi=0.01$. With the in-plane inertia/smaller damping, the response shows unstable nature after 


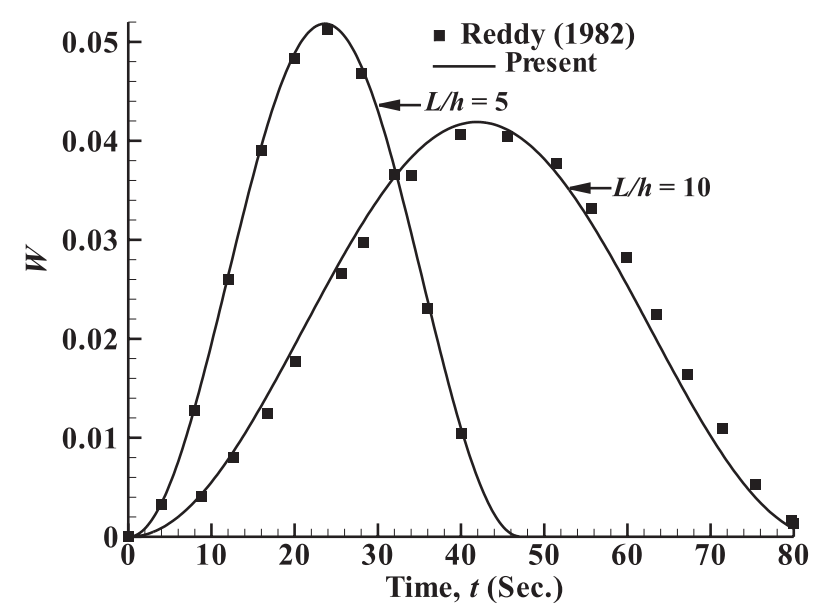

Figure 4. Comparison of transverse displacement history of two-layered cross-ply $\left(0^{\circ} / 90^{\circ}\right)$ square bimodular laminate $(L / b=1)$.

few cycles. This numerical instability in the direct time integration approach is due to the sudden change in the restoring force $([\mathbf{K}]\{\boldsymbol{\delta}\})$ when response changes from positive/negative half cycle to negative/positive half cycle. The abrupt change in restoring force excites higher
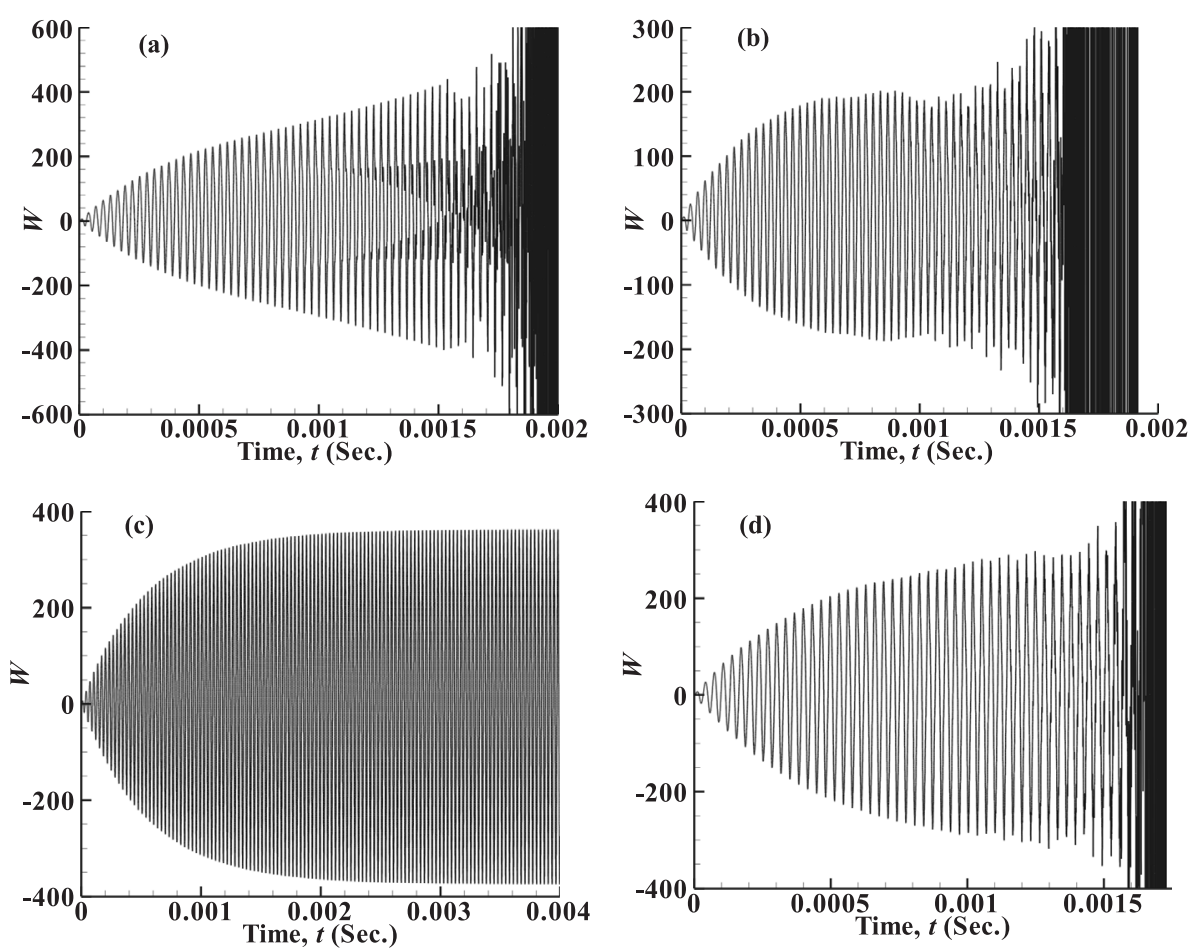

Figure 5. Non-dimensional transverse central displacement $(W)$ history of eight-layered bimodular CSCS panel $\left(L / b=0.5, b / h=10, r / h=50, E_{2 t} / E_{2 c}=0.2, \omega_{F} / \omega=1, \xi=0.01\right.$, $\left.\Delta t=\pi / 200 \omega_{F}\right)$ : (a) $\left(0^{\circ} / 90^{\circ}\right)_{4}$, without in-plane inertia, (b) $\left(0^{\circ} / 90^{\circ}\right)_{4}$, with in-plane inertia, (c) $\left(45^{\circ} /-45^{\circ}\right)_{4}$, without in-plane inertia, (d) $\left(45^{\circ} /-45^{\circ}\right)_{4}$, with in-plane inertia. 

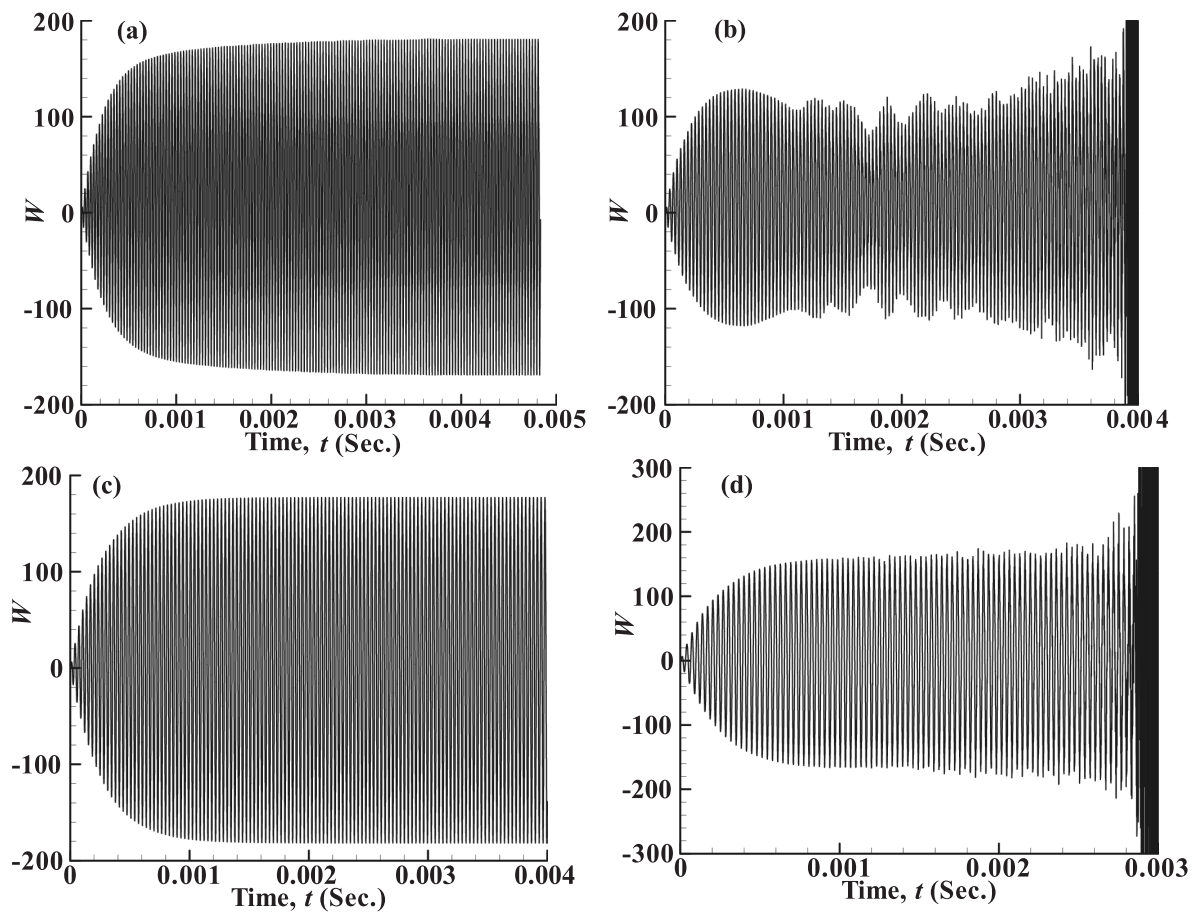

Figure 6. Non-dimensional transverse central displacement $(W)$ history of eight-layered bimodular CSCS panel $\left(L / b=0 \cdot 5, b / h=10, r / h=50, E_{2 t} / E_{2 c}=0 \cdot 2, \omega_{F} / \omega=1, \xi=0 \cdot 02\right.$, $\left.\Delta t=\pi / 400 \omega_{F}\right)$ : (a) $\left(0^{\circ} / 90^{\circ}\right)_{4}$, without in-plane inertia, (b) $\left(0^{\circ} / 90^{\circ}\right)_{4}$, with in-plane inertia, (c) $\left(45^{\circ} /-45^{\circ}\right)_{4}$, without in-plane inertia, (d) $\left(45^{\circ} /-45^{\circ}\right)_{4}$, with in-plane inertia.

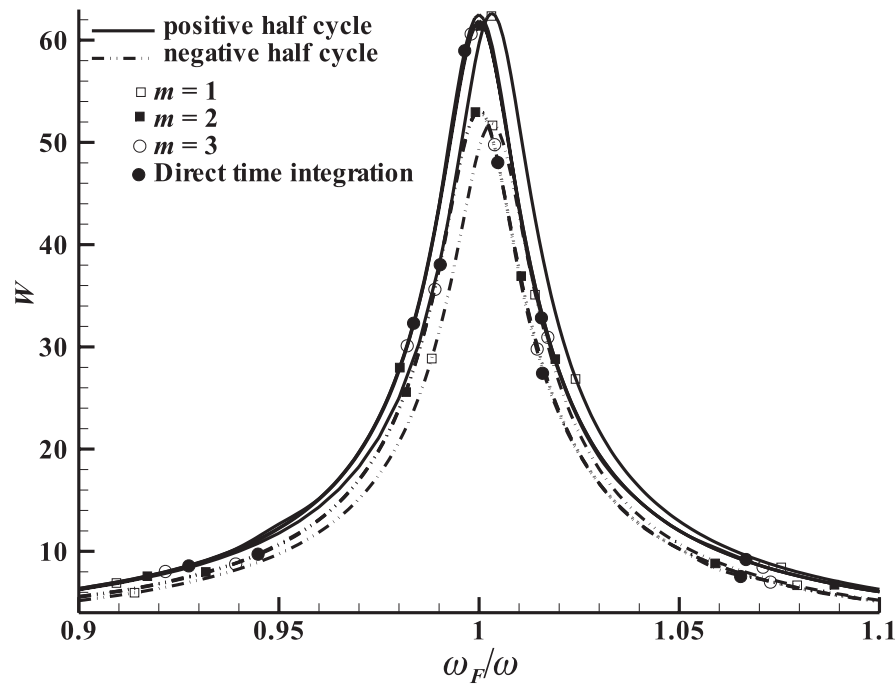

Figure 7. Convergence study for frequency response of two-layered cross-ply $\left(0^{\circ} / 90^{\circ}\right) \mathrm{CCCC}$ cylindrical panel $\left(L / b=1, b / h=10, r / h=100, E_{2 t} / E_{2 c}=0 \cdot 2, \xi=0 \cdot 01\right)$ without in-plane inertia. 


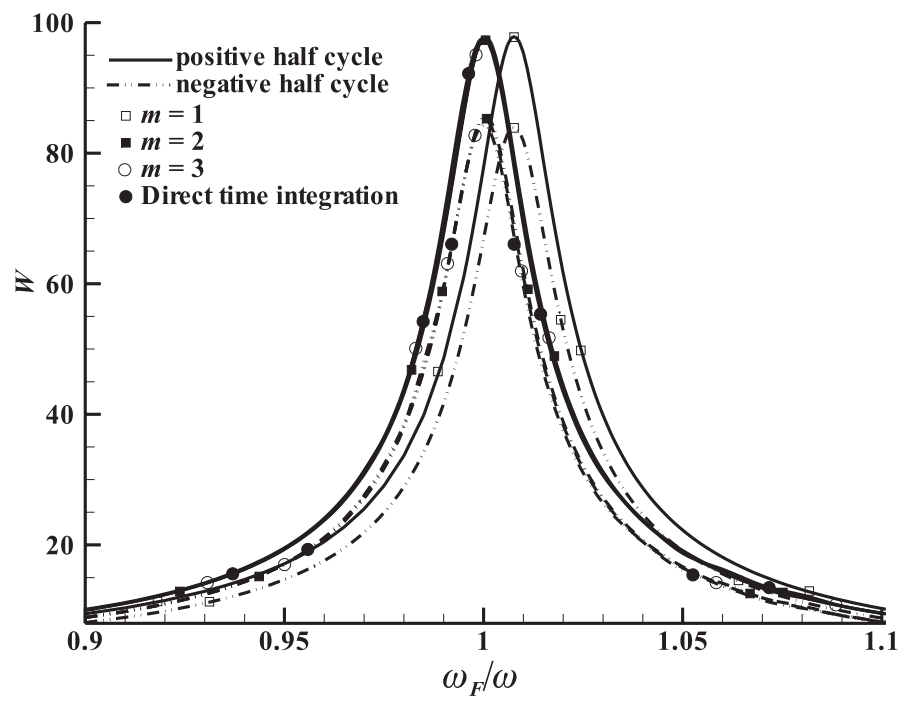

Figure 8. Convergence study for frequency response of two-layered cross-ply $\left(0^{\circ} / 90^{\circ}\right) \mathrm{CSCS}$ cylindrical panel $\left(L / b=1, b / h=10, r / h=100, E_{2 t} / E_{2 c}=0 \cdot 2, \xi=0 \cdot 01\right)$ without in-plane inertia.

harmonics at every instant of a cycle change leading to numerical instability. The study with smaller time steps (results are not presented for the sake of brevity) shows that the numerical instability can not be eliminated even for very small time increments $\left(\Delta t=\pi / 3000 \omega_{F}\right)$. Therefore, the detailed study of frequency response of bimodular cylindrical panels is carried out using Galerkin method based approach.

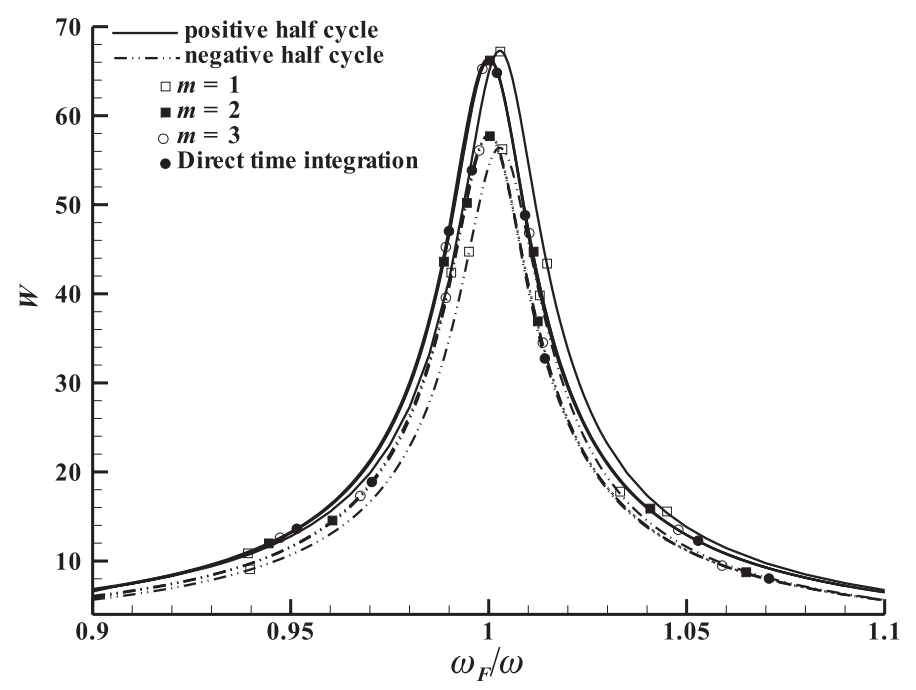

Figure 9. Convergence study for frequency response of eight-layered angle-ply $\left(45^{\circ} /-45^{\circ}\right)_{4} \mathrm{CCCC}$ cylindrical panel $\left(L / b=1, b / h=10, r / h=100, E_{2 t} / E_{2 c}=0 \cdot 2, \xi=0 \cdot 01\right)$ without in-plane inertia. 


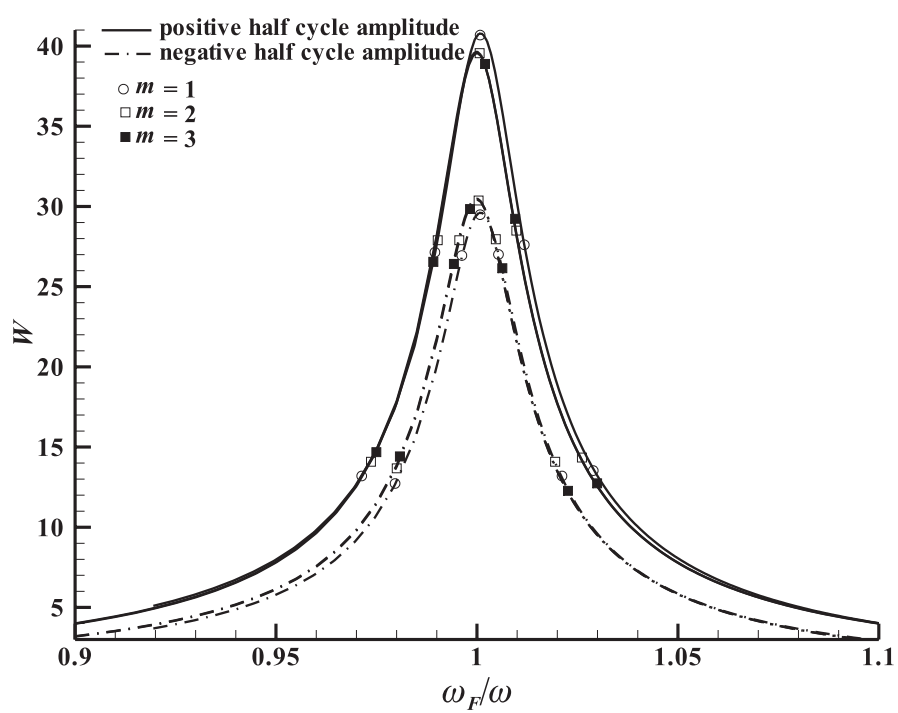

Figure 10. Convergence study for frequency response of eight-layered angle-ply $\left(0^{\circ} / 90^{\circ}\right)_{4} \mathrm{CCCC}$ cylindrical panel $\left(L / b=1, b / h=10, r / h=50, E_{2 t} / E_{2 c}=0 \cdot 2, \xi=0 \cdot 01\right)$ with in-plane inertia.

The convergence study of frequency response with number of terms $(m)$ of Galerkin solution (equation 9) for CCCC, CSCS cross-ply $\left(0^{\circ} / 90^{\circ}\right)$ and CCCC angle-ply $\left(45^{\circ} /-45^{\circ}\right)_{4}$ panels $\left(L / b=1 \cdot 0, b / h=10, r / h=100, E_{2 t} / E_{2 c}=0 \cdot 2, \xi=0 \cdot 01\right)$ without considering inplane inertia is given in figures 7-9. The results obtained using direct time integration are also given for comparison purpose. It can be seen from these figures that two-term solution $(m=2)$ yields accurate frequency response but one-term solution $(m=1)$ is not sufficient

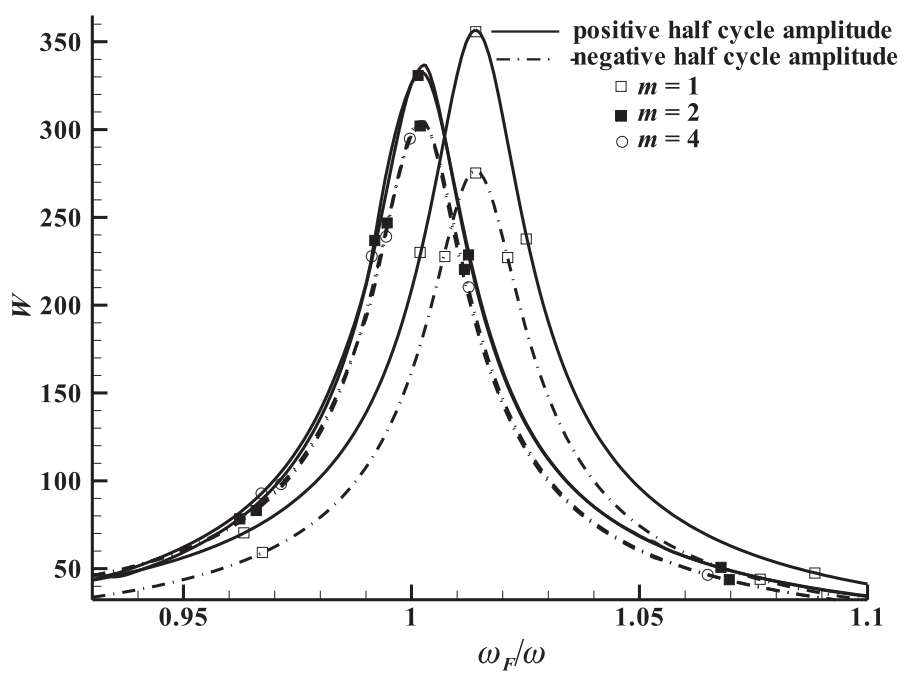

Figure 11. Convergence study for frequency response of eight-layered cross-ply $\left(0^{\circ} / 90^{\circ}\right)_{4} \mathrm{CSCS}$ cylindrical panel $\left(L / b=0 \cdot 5, b / h=10, r / h=50, E_{2 t} / E_{2 c}=0 \cdot 2, \xi=0 \cdot 01\right)$ with in-plane inertia. 

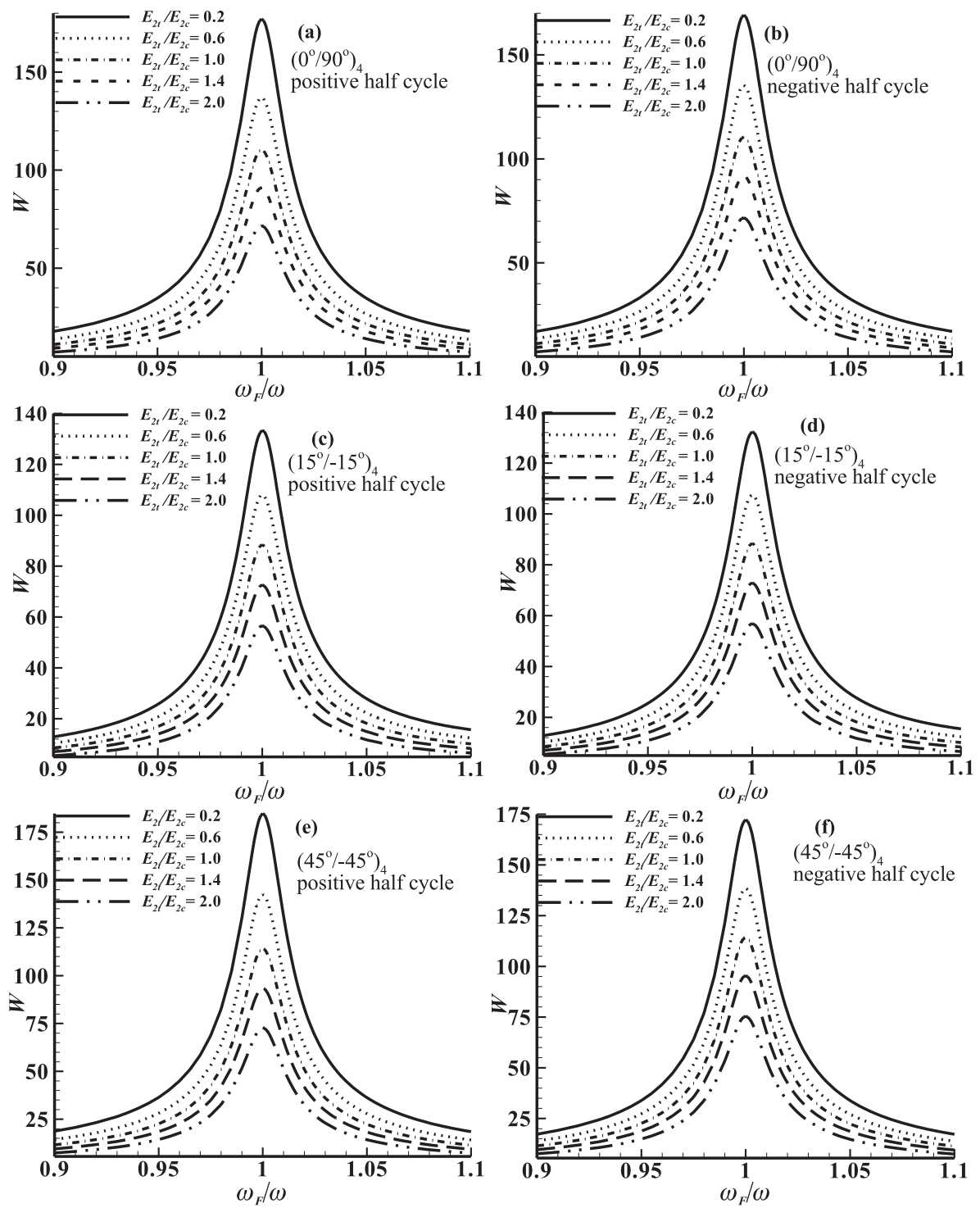

Figure 12. Frequency response of eight-layered CCCC panels $(L / b=0 \cdot 5, b / h=10, r / h=100$, $\xi=0.01)$ with in-plane inertia for different $E_{2 t} / E_{2 c}$ ratios.

for accurate results. Further, the converged frequency response curves are in good agreement with those obtained using direct time integration. The convergence study for typical case of CCCC panel $\left[L / b=1 \cdot 0, b / h=10, r / h=50,\left(0^{\circ} / 90^{\circ}\right)_{4}, E_{2 t} / E_{2 c}=0 \cdot 2, \xi=0.01\right]$ and CSCS panel $\left[L / b=0.5, b / h=10, r / h=50,\left(0^{\circ} / 90^{\circ}\right)_{4}, E_{2 t} / E_{2 c}=0 \cdot 2, \xi=0.01\right]$ considering in-plane inertia is given in figures 10 and 11 , respectively. It can be seen that the two-term solution $(m=2)$ gives accurate results for both positive and negative half cycle amplitudes.

The frequency responses of eight-layered cross- $\left(0^{\circ} / 90^{\circ}\right)_{4}$ and angle-ply $\left[\left(15^{\circ} /-15^{\circ}\right)_{4}\right.$, $\left(45^{\circ} /-45^{\circ}\right)_{4}$ ] CCCC cylindrical panels $(L / b=0.5, r / h=100, b / h=10, \xi=0.01)$ 

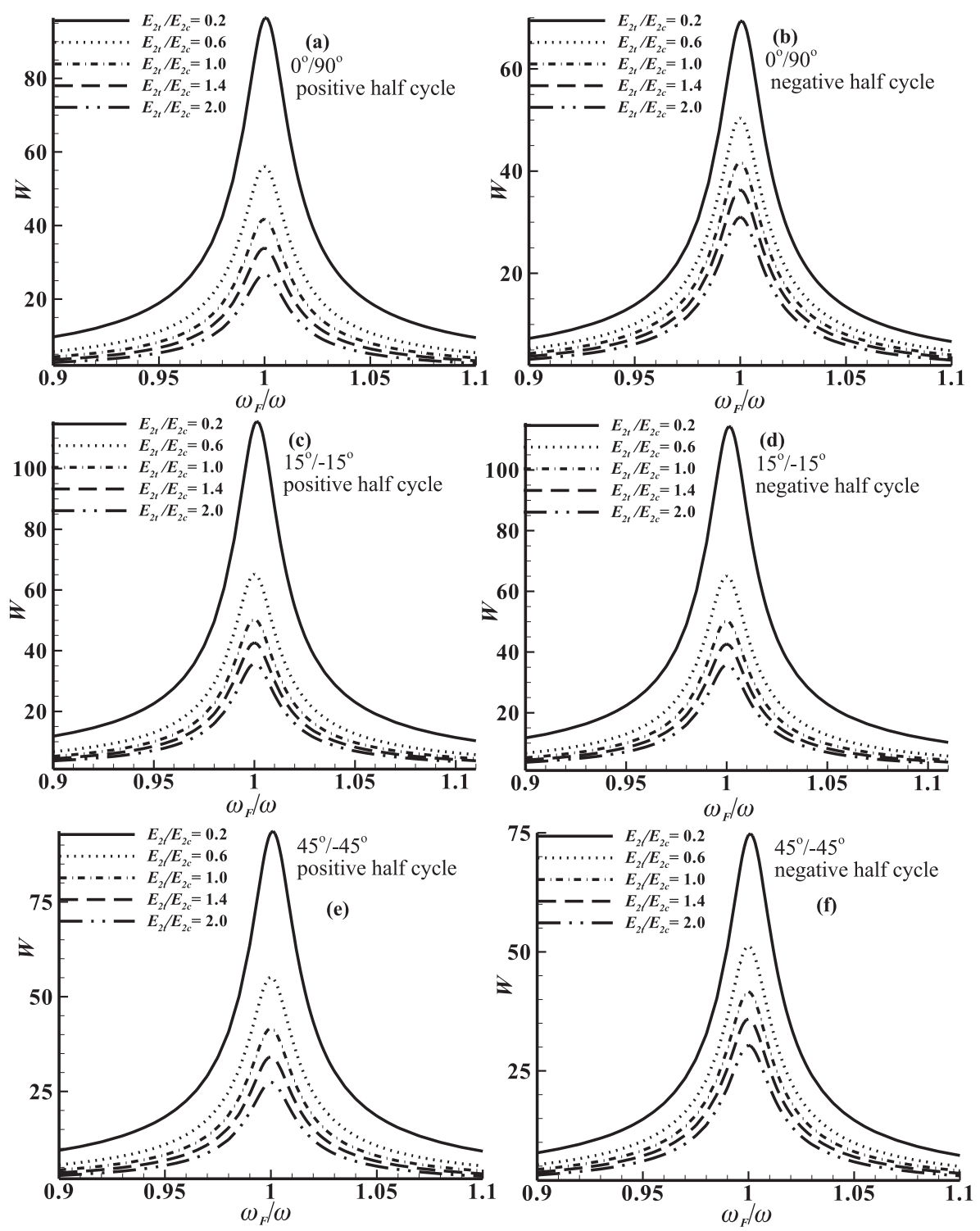

Figure 13. Frequency response of two-layered CSCS panels $(L / b=1 \cdot 0, b / h=10, r / h=50$, $\xi=0.01$ ) with in-plane inertia for different $E_{2 t} / E_{2 c}$ ratios.

considering in-plane inertia are shown in figure 12 for $E_{2 t} / E_{2 c}=0 \cdot 2,0 \cdot 6,1 \cdot 0,1.4$ and 2.0. It can be seen from this figure that with the increase in $E_{2 t} / E_{2 c}$ ratio, the positive and negative half cycle amplitudes of central transverse displacement $(W)$ decrease. The positive half cycle amplitude is greater than the negative half cycle amplitude for $E_{2 t} / E_{2 c}<1$ and vice-versa for $E_{2 t} / E_{2 c}<1$. The percentage difference of positive and negative half cycle amplitudes decreases with the increase in $E_{2 t} / E_{2 c}$ from 0.2 to 2 . Further, the response amplitude of $\left(15^{\circ} /-15^{\circ}\right)_{4}$ panels is significantly less than that of $\left(0^{\circ} / 90^{\circ}\right)_{4}$ and $\left(45^{\circ} /-45^{\circ}\right)_{4}$ panels. The frequency responses of two-layered cross- $\left(0^{\circ} / 90^{\circ}\right)$ and angle-ply $\left[\left(15^{\circ} /-15^{\circ}\right)\right.$, 


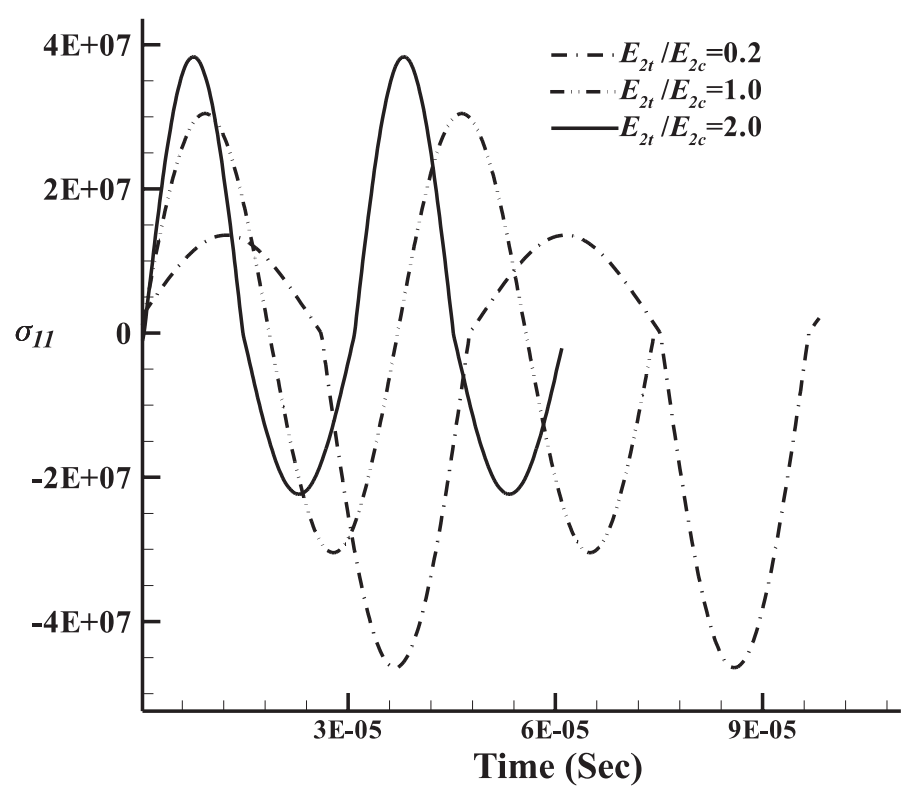

Figure 14. In-plane normal stress ( $\sigma_{11}$ at top centre of the laminate) versus time for two-layered cross-ply CCCC cylindrical panel $\left(r / h=50, b / h=10, L / b=1,0^{\circ} / 90^{\circ}, \omega_{F} / \omega=1, \xi=0 \cdot 01\right)$.

$\left.\left(45^{\circ} /-45^{\circ}\right)\right]$ CSCS cylindrical panels $(L / b=1, r / h=50, b / h=10, \xi=0 \cdot 01)$ considering in-plane inertia are shown in figure 13. It can be observed from this figure that the response amplitude of $\left(15^{\circ} /-15^{\circ}\right)$ panels is more than that of $\left(0^{\circ} / 90^{\circ}\right)$ and $\left(45^{\circ} /-45^{\circ}\right)$ panels. The percentage difference of positive and negative half cycle amplitudes is also more for $\left(0^{\circ} / 90^{\circ}\right)$ and $\left(45^{\circ} /-45^{\circ}\right) \mathrm{CSCS}$ panels compared to the case of CCCC panels.

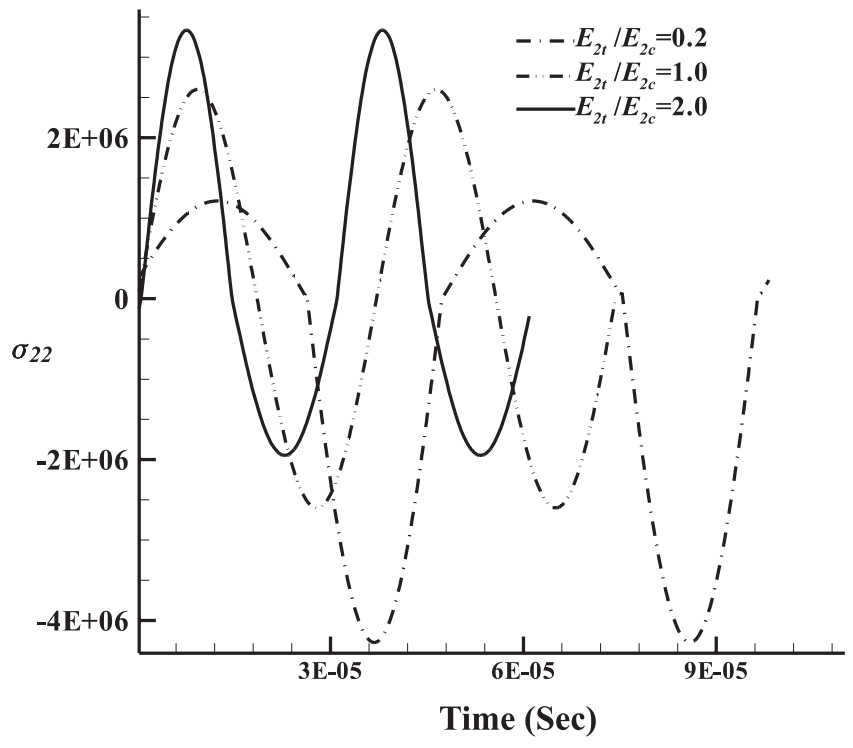

Figure 15. In-plane normal stress $\left(\sigma_{22}\right.$ at top centre of the laminate) versus time for two-layered cross-ply CCCC cylindrical panel $(r / h=50, b / h=10, L / b=1$, $\left.0^{\circ} / 90^{\circ}, \omega_{F} / \omega=1, \xi=0 \cdot 01\right)$. 


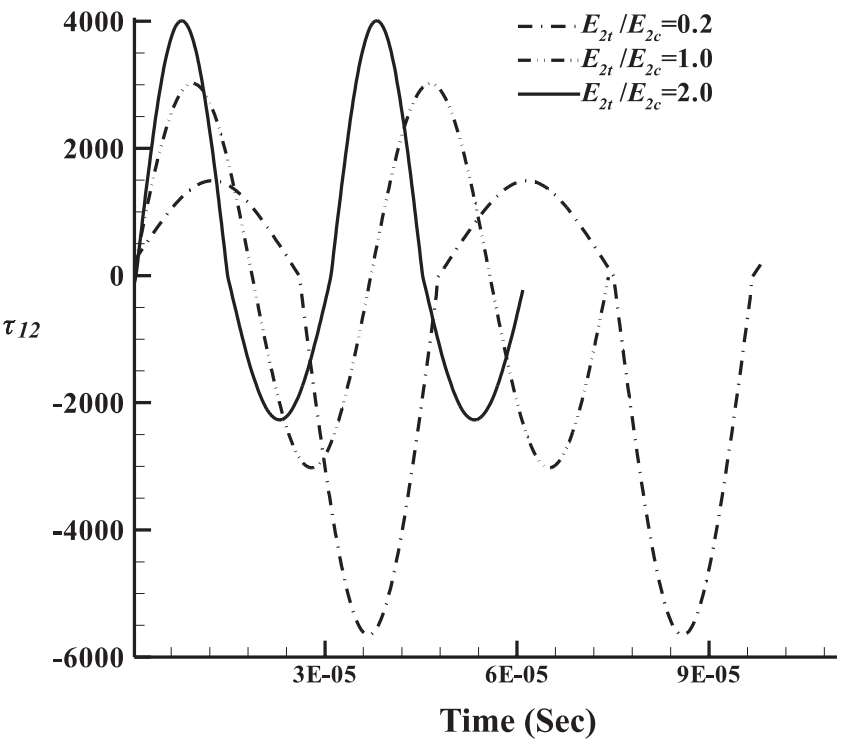

Figure 16. In-plane shear stress $\left(\tau_{12}\right.$ at top centre of the laminate) versus time for two-layered cross-ply CCCC cylindrical panel $(r / h=50, b / h=10, L / b=1$, $\left.0^{\circ} / 90^{\circ}, \omega_{F} / \omega=1, \xi=0 \cdot 01\right)$.

The in-plane stresses at top centre $(s=L / 2, \theta=\Psi / 2, z=h / 2)$ of cylindrical panel $\left(r / h=50, L / b=1, b / h=10,0^{\circ} / 90^{\circ}, q_{0}=100000 \mathrm{~N} / \mathrm{m}^{2}\right)$ are shown in figures 1416 for two cycles. The magnitude of negative half cycle stresses are significantly greater compared to positive half cycle stresses for $E_{2 t} / E_{2 c}=0 \cdot 2$ and vice versa for $E_{2 t} / E_{2 c}=2$. The magnitude of positive half cycle stress is not same as magnitude of negative half cycle stress. For unimodular material $\left(E_{2 t} / E_{2 c}=1\right)$, the positive and negative half cycle stresses are same.

\section{Conclusions}

The effect of bimodularity ratio on the frequency response of cross- and angle-ply laminated cylindrical panels is studied using finite element method based on first order shear deformation theory and Bert's constitutive model. The bimodularity ratio is varied so as to cover the materials having tensile properties greater than compressive one and vice versa. The solution of governing equations is obtained using direct time integration and Galerkin approach. It is found that for some parameters of bimodulus panels, the direct time integration approach fails to predict steady state response due to numerical instability. Galerkin's based approach is found suitable for frequency response analysis of bimodular laminated panels. The following conclusions can be drawn from the study:

(i) Two-term Galerkin solution is necessary for converged results for the cases considered. (ii) With the increase in $E_{2 t} / E_{2 c}$ ratio, the positive and negative half cycle amplitudes of central transverse displacement $(W)$ decrease. (iii) The positive half cycle amplitude is greater than the negative half cycle amplitude for $E_{2 t} / E_{2 c}<1$ and is smaller for $E_{2 t} / E_{2 c}<1$. (iv) The percentage difference of positive and negative half cycle amplitudes decreases with the increase in $E_{2 t} / E_{2 c}$. (v) The stresses for positive half and negative half of a vibration cycle are significantly different. 
The financial support received from Council of Scientific and Industrial Research (CSIR), New Delhi (India) through Grant No. 22(0401)/06/EMR-II is gratefully acknowledged.

\section{Nomenclature}

$\begin{array}{ll}b & \text { Circumferential length } \\ \beta & \text { Damping coefficient } \\ \beta_{s}, \beta_{\theta} & \text { Rotations of the meridional and circumferential sections } \\ {[\mathrm{C}]} & \text { Damping matrix } \\ \{\delta\} & \text { Degrees of freedom vector } \\ E_{1 t}, E_{1 c} & \text { Longititudinal Young's moduli in tension and compression } \\ E_{2 t}, E_{3 t} & \text { Transverse Young's moduli in tension } \\ E_{2 c}, E_{3 c} & \text { Transverse Young's moduli in compression } \\ \{\varepsilon\} & \text { Strain vector } \\ \left\{\varepsilon_{p}\right\} & \text { Membrane strain vector } \\ \left\{\varepsilon_{b}\right\} & \text { Bending strain vector } \\ \left\{\varepsilon_{s}\right\} & \text { Transverse shear strain vector } \\ G_{12 t}, G_{13 t}, G_{23 t} & \text { In-plane, longitudinal-thickness and transverse-thickness shear moduli in } \\ G_{12 c}, G_{13 c}, G_{23 c} & \text { In-plane, longitudinal-thickness and transverse-thickness shear moduli in } \\ h & \quad \text { compression } \\ {[\mathrm{K}]} & \text { Total thickness of laminate } \\ {\left[\mathrm{K}_{1}\right]} & \text { Stiffness matrix } \\ {\left[\mathrm{K}_{2}\right]} & \text { Stiffness matrix for positive half cycle } \\ L & \text { Stiffness matrix for negative half cycle } \\ {[\mathrm{M}]} & \text { Meridional length } \\ N & \text { Mass matrix } \\ \xi & \text { Number of layers } \\ {\left[\bar{Q}_{i j k l}\right]} & \text { Modal damping factor } \\ q_{0} & \text { Reduced stiffness coefficient matrix } \\ \{\mathrm{R}\} & \text { Uniformly distributed load magnitude } \\ r & \text { Residual force vector } \\ \rho & \text { Radius of middle surface of laminated panel } \\ \left\{\sigma^{k}\right\} & \text { Density } \\ s, \theta, z & \text { stress vector of } k^{\text {th }} \text { layer } \\ \sigma_{11} & \text { Meridional, circumferential and thickness coordinates } \\ \sigma_{22} & \text { Fibre direction normal stress } \\ T & \text { Transverse to fibre direction normal stress } \\ \tau_{12} & \text { Kinetic energy } \\ U & \text { In-plane shear stress } \\ u, v, w & \text { Total potential energy } \\ u_{0}, v_{0}, w_{0} & \text { Displacement in the meridional, circumferential and thickness directions } \\ v_{12 t}, v_{13 t}, v_{23 t} & \text { Mid-surface displacements in the meridional, circumferential and } \\ v_{12 c}, v_{13 c}, v_{23 c} & \text { thickness directions } \\ & \text { in-plane, longitudinal-thickness and transverse-thickness Poission's ratio } \\ & \text { in compression } \\ & \end{array}$




$\begin{array}{ll}\Psi & \text { Sector angle } \\ W & \text { Non-dimensional transverse displacement } \\ \omega_{1}, \omega_{2} & \text { Positive and negative half cycle natural frequencies } \\ \omega & \text { Average natural frequency } \\ \omega_{F} & \text { Forcing frequency }\end{array}$

\section{References}

Bathe K J 1996 Finite element procedures (New Jersey: Prentice-Hall)

Bert C W 1977 Models for fibrous composites with different properties in tension and compression. J. Eng. Materials and Technol. Trans. ASME 99H: 344-349

Bert C W, Reddy J N, Chao W C, Reddy V S 1981 Vibration of thick rectangular plates of bimodulus composite material. ASME J. Appl. Mech. 48: 371-376

Bert C W, Kumar M 1982 Vibration of cylindrical shells of bimodulus composite material. J. Sound Vib. 81: 107-121

Doong J L, Chen L W 1985 Vibration of a bimodulus thick plate. ASME J. Vibration, Acoustics, Stress and Reliability in Design 107: 92-97

Doong J L, Fung C P 1988 Vibration and buckling of bimodulus laminated plates according to a higher-order plate theory. J. Sound Vib. 125: 325-339

Khan K, Patel B P, Nath Y 2007 Free vibration of bimodulus laminated angle-ply cylindrical panels, Proceedings of the $4^{\text {th }}$ International Conference on Theoritical, Applied, Computational and Experimental Mechanics ICTACEM 07, December 27-29, 2007, IIT Kharagpur, India

Khan K, Patel B P, Nath Y 2008 Free vibration of bimodulus laminated cross-ply conical panels, Proceedings of the $9^{\text {th }}$ Biennial ASME conference on Engineering System Design and Analysis ESDA08, July 7-9, 2008, Haifa, Israel

Khan K, Patel B P, Nath Y 2009a Vibration analysis of bimodulus laminated cylindrical panels. J. Sound Vib. 321: 166-183

Khan K, Patel B P, Nath Y 2009b Frequency response of bimodular cross-ply laminated cylindrical panels. J. Sound Vib. 327: 55-69

Kraus H 1976 Thin elastic shells (New York: John Willy)

Patel B P, Gupta S S, Sarda R 2005a Free flexural vibration behaviour of bimodular material angle-ply laminated composite plates. J. Sound Vib. 286: 167-186

Patel B P, Gupta S S, Joshi M, Ganapathi M 2005b Transient response analysis of bimodulus aniosotropic laminated composite plates. J. Reinforced Plastics and Composites 24: 795-821

Prathap G, Naganarayana B P, Somasekhar B R 1988 Field consistency analysis of the isoparametric eight-noded plate bending element. Computers and Struct. 29: 857-863

Reddy J N 1982 Transient response of laminated bimodular-material composite rectangular plates. J. Composite Materials 16: 139-152

Venkatrao M, Mahajan P, Mittal R K 2008 Effect of architecture on mechanical properties of carbon/carbon composites. Composite Struct. 83: 131-142 